\title{
Interaction Between Oxygen Interstitials and Deformation Twins in alpha-Titanium
}

\author{
W J Joost ${ }^{1, *}$, S Ankem ${ }^{1}$, and M M Kuklja ${ }^{1}$ \\ ${ }^{1}$ University of Maryland, Department of Materials Science and \\ Engineering, College Park, Maryland 20782, USA \\ *wjoost@umd.edu
}




\begin{abstract}
Twinning is an important deformation mechanism in many hexagonal close packed metals, including $\alpha$-titanium (Ti) alloys. However, the mechanisms for twin nucleation, growth, and interaction with other defects are not completely understood. In this study we interrogate the behavior of oxygen $(\mathrm{O})$ interstitials near a $(10 \overline{1} 2)$ twin boundary using a combination of density functional theory (DFT) and modified embedded atom method (MEAM) calculations. The presence of the twin boundary significantly affects both interstitial formation energy as well as the activation barriers for diffusion between sites. We demonstrate that a tetrahedral interstitial is stable in the twin boundary, despite being unstable in bulk Ti, while the formation energies of the octahedral, hexahedral, and crowdion interstitials are all modified by a nearby twin. Further, the activation barriers for diffusion in the region near the twin are uniformly lower than in the bulk. An atom diffusing across the twin boundary moves through several paths with peak energies more than $0.3 \mathrm{eV}$ lower than for comparable diffusion far from the twin, suggesting that the (1012) twin is a fast diffusion pathway and movement of oxygen interstitials across the twin during twin growth is possible.
\end{abstract}

Keywords: Titanium, Oxygen, Twinning, Diffusion, Empirical Potentials 


\section{Introduction}

Titanium (Ti) alloys exhibit excellent specific strength, toughness, high temperature stability, and corrosion resistance, among other characteristics [1]. This unique set of properties enables use in applications as varied as jet engines [2], artificial hip joints [3], offshore oil drilling equipment [4], and suspension components for the Spirit and Opportunity Mars rovers [5]. Deformation of Ti alloys, whether hexagonal close packed (hcp) $\alpha$-phase, body centered cubic (bcc) $\beta$-phase, or a combination thereof, can occur via twinning; understanding the effects of alloy chemistry and impurity content on deformation twinning is therefore an important step towards improved mechanical performance and reliability.

Oxygen (O) has up to 33 at\% solubility in $\alpha$-Ti [6] and is a common impurity and alloying element. Interstitial $\mathrm{O}$ is also believed to interact with deformation twins, and may result in time-dependent twinning by obstructing the shearing/shuffling process associated with twin growth [7]. Early work by Biget \& Saada identified that the shuffle distances for interstitial impurities in Zr (an hcp metal with a simi-

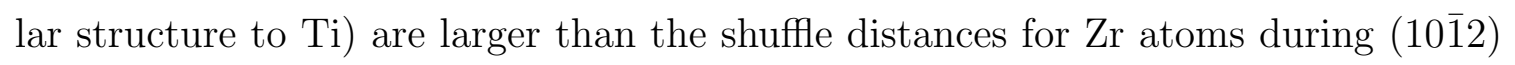
and $(11 \overline{2} 1)$ twinning, although the possibility for O-Zr interference during shuffling was not described [8]. More recent experimental measurements combined with a crystallographic model suggest that $\mathrm{O}$ interstitial sites interfere with the shuffle of Ti atoms during twin growth in body centered cubic (bcc) Ti, requiring $\mathrm{O}$ interstitial diffusion away from or across the twin to enable further twin growth [9]. In addition, a decrease in (1012) twin growth rate during creep in $\alpha$-Ti may be associated with the presence of $\mathrm{O}$ interstitials based on a similar crystallographic model 
[10, 11]. The width of $(10 \overline{1} 2)$ twins decreases with increasing strain rate during quasi-static loading, providing further evidence of time dependence of twin growth in $\alpha$-Ti [12]. The measured activation energy for twin growth in $\alpha$-Ti, calculated using the diffusion coefficient for $\mathrm{O}$ diffusion in the bulk, is approximately $0.684 \mathrm{eV}$ during creep loading [11]; the activation energy for $\mathrm{O}$ diffusion in bulk $\alpha$-Ti was recently measured as $1.75 \pm 0.52 \mathrm{eV}$ using high purity samples and the nuclear resonance technique [13], while DFT calculations yield an activation barrier of $2.08 \mathrm{eV}$ [14]. Historical measurements with reasonable results indicate activation energy for diffusion between $1.45 \mathrm{eV}$ and $2.68 \mathrm{eV}$ [15]. Should $\mathrm{O}$ interstitials interact with twin growth as crystallographic modeling suggests, the difference between the bulk diffusion activation barrier and the activation barrier for twin growth indicates that twin growth and $\mathrm{O}$ diffusion mechanisms interact in a complicated way. The length and time scales of these interactions make experimental measurement very difficult, while atomistic modeling provides an excellent tool set for gaining an improved, mechanistic understanding of the processes. Density functional theory (DFT) calculations by Ghazisaeidi and Trinkle demonstrate that a (1012) twin boundary affects the formation energy of octahedral oxygen interstitials in the immediate vicinity of the twin [16], however the effect of the twin on formation energy of other sites and the activation barriers for diffusion between the sites is not known. Recent DFT calculations reveal that $\mathrm{O}$ diffuses readily along the core of a prismatic edge dislocation in $\alpha$-Ti and that the presence of $\mathrm{O}$ increases Peierls stress and modifies dislocation core geometry [17], providing further indication of complicated interactions between Ti deformation mechanisms and $\mathrm{O}$ interstitials. In this study we interrogate the 


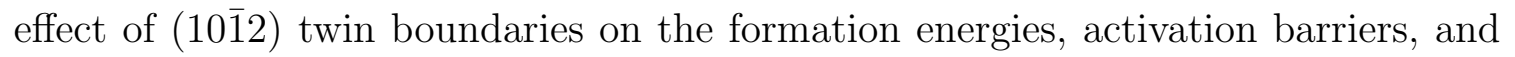
diffusion pathways for O. We report that the presence of a (101̄2) twin boundary has a significant effect on the thermodynamics and kinetics of nearby $\mathrm{O}$ interstitials.

\section{Computational Techniques}

Investigating material behavior often requires multiple computational techniques to properly address the range of relevant time and length scales. Directly simulating interactions of $\mathrm{O}$ defects with a crystallographically complicated planar defect such as a twin requires atomistic modeling, however many such techniques exist. In this study, we employ a combination of DFT and modified embedded atom method (MEAM) calculations to leverage the benefits and offset the disadvantages of each tool. DFT is an ab-initio technique that offers excellent predictive power but is limited to simulation of relatively few atoms for relatively brief periods. The MEAM is an empirical potential method that trades a loss of rigorous physical modeling for capability to simulate substantially larger systems for longer periods of time when compared to DFT. Our DFT calculations are performed in the Vienna Ab-initio Simulation Package (VASP) [18, 19, 20, 21], employing projector augmented wave (PAW) psuedopotentials [22, 23] and the Perdew-Burke-Ernzerhof generalized gradient approximation (GGA-PBE) [24]. The cut-off energy for all calculations was held at $520 \mathrm{eV}$. We apply Methfessel-Paxton smearing of $0.2 \mathrm{eV}$ and use a $2 \times 1 \times 6$ Monkhorst-Pack $k$-point mesh. Relaxation calculations were stopped when the force on each atom in the system was $<5 \mathrm{meV} / \AA$. Our MEAM calculations are performed 
in LAMMPS [25] using published MEAM potentials for Ti [26], O [27], and Ti-O [28], noting that the Ti-O potential was fit specifically to study the interaction between O interstitials and twins in Ti alloys. Among several variations of the MEAM, we use the second nearest neighbor $(2 \mathrm{NN})$ technique as outlined by Lee et al. [29, 30], following the earlier work by Baskes [31, 32, 33, 34]. Activation barriers for diffusion are calculated using the climbing imagine nudged elastic band (CI-NEB) approach with three to seven intermediate images [35, 36, 37]. Creation of the supercells for these calculations, visualization of results, and production of several figures was accomplished using the Open Visualization Tool (OVITO) [38]. The data used to produce Figures 5, 7, and 8 are archived and available [39].

\section{Structural Model}

\section{1 (10̄̄2) Twin in Pure Ti}

We produced several supercells in order to investigate of the effects of distance between twins and oxygen interstitials on the behavior of the system. Figure 1 shows our "short" and "tall" twin supercells, which differ in size along the $y$-direction, along with the coordinate system used in this study. Each cell contains two (10 $\overline{1} 2)$ twin boundaries as required in order to maintain periodicity in the $y$-direction. Cell depth in the $z$-direction (which corresponds to [1210]) varies in intervals of the lattice

parameter of $\alpha$-Ti, $a$, which is equal to $2.95 \AA$ [40]. To create a supercell containing a $(10 \overline{1} 2)$ twin we first rotate the hcp titanium unit cell such that the $[1 \overline{2} 10]$ direction is aligned with the $z$-direction. One side of the twin is rotated so that the normal 
to the (1012) plane is aligned with the $y$-direction, while the second side of the twin is rotated so that the normal to the $(\overline{1} 012)$ is aligned with the $y$-direction. Volumes of atoms rotated for each side of the twin are combined into a single supercell and trimmed in the $x$ - and $z$-directions for periodicity. Relaxation of the initial twin structure is accomplished in two steps: first, the cell is relaxed in the $y$-direction only while the atoms are allowed to relax completely, after which the cell and atoms are free to relax in all directions, although the cell is prevented from shear distortion.

We first compare the experimentally measured twin boundary structure [41] with the results of our DFT and MEAM calculations with $z=2 a$. Figure 2 compares the DFT, MEAM, and experimental structures, with the structure motif superimposed for clarity; the calculated (10̄12) structures relax to a planar twin as found experimentally, while the angle between basal planes across the twin boundary, $\theta$, is also correctly captured. The $(10 \overline{1} 2)$ twin energy is $0.301 \mathrm{~J} / \mathrm{m}^{2}$ calculated using DFT, in good agreement with previous reports [42, 16], and $0.410 \mathrm{~J} / \mathrm{m}^{2}$ calculated using the MEAM. The relaxed structure and twin boundary energy is identical for the short and tall twin supercells and is not affected by changes to supercell depth in the $x$ - or $z$-directions. While the MEAM overestimates the twin boundary energy by comparison to DFT, the formation energies of the many oxygen interstitials studied here are not significantly affected.

\subsection{Octahedral O Interstitials Near a (10̄̄2) Twin}

Having obtained the correct $(10 \overline{1} 2)$ twin structure for pure $\alpha$-Ti, we calculate the formation energy of octahedral O interstitials near the twin boundary following ear- 


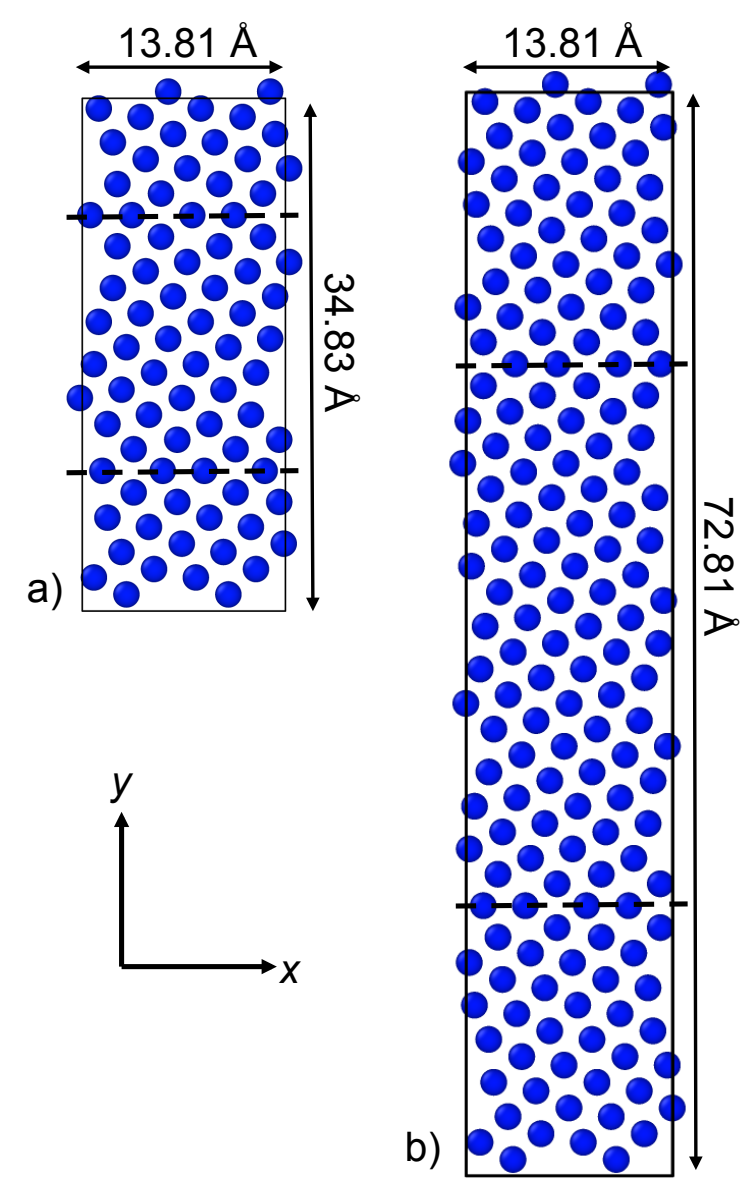

Figure 1: Front view of the a) "short" and b) "tall" twin supercells along with the coordinate system used in this study. The twin boundaries are indicated with dashed lines. The supercells vary in depth along the $z$-direction (out of the page), which corresponds to $[1 \overline{2} 10]$. 


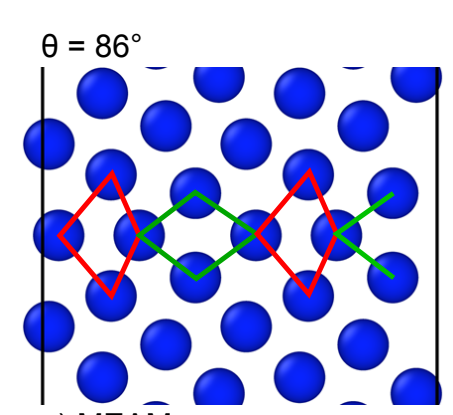

a) MEA $\bar{M}$

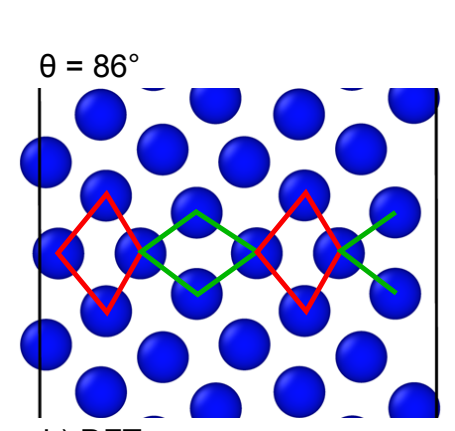

b) DFT

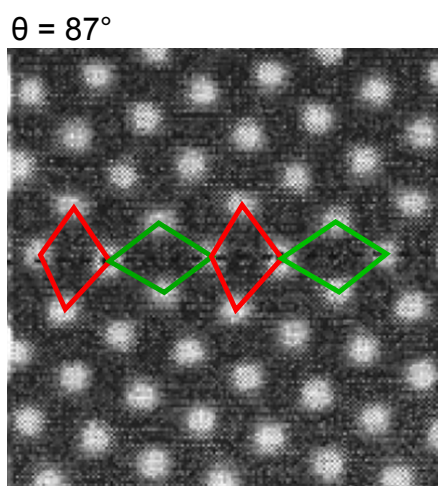

c) Experiment (HR-TEM)

Figure 2: Relaxed (1012) twin structure a) calculated using the MEAM, b) calculated using DFT, and c) measured experimentally using high-resolution transmission electron microscopy (HR-TEM) [41]. The angle between basal planes across the twin boundary, $\theta$, is also reported.

lier work by Ghazisaeidi and Trinkle [16], employing our short supercell and their notation for the numbering of the octahedral sites. The five octahderal interstitial sites studied here are shown in Figure 3. Formation energy of the interstitials near the twin is calculated relative to the energy of the system with an interstitial at site O0, where the cell size for calculation of all interstitials is fixed at the fully relaxed size with an interstitial at site O0. As shown in Table 1, despite a different supercell size and slightly different DFT calculation settings, our results are quite similar. The MEAM potential predicts formation energy within $0.2 \mathrm{eV}$ of the DFT calculated results for each octahedral interstitial.

A benefit of applying the MEAM is the capacity for large supercells at minimal computational cost. Hence while our DFT calculations are limited to the short twin supercell with a depth of $5.90 \AA$, we can explore much larger systems using the 


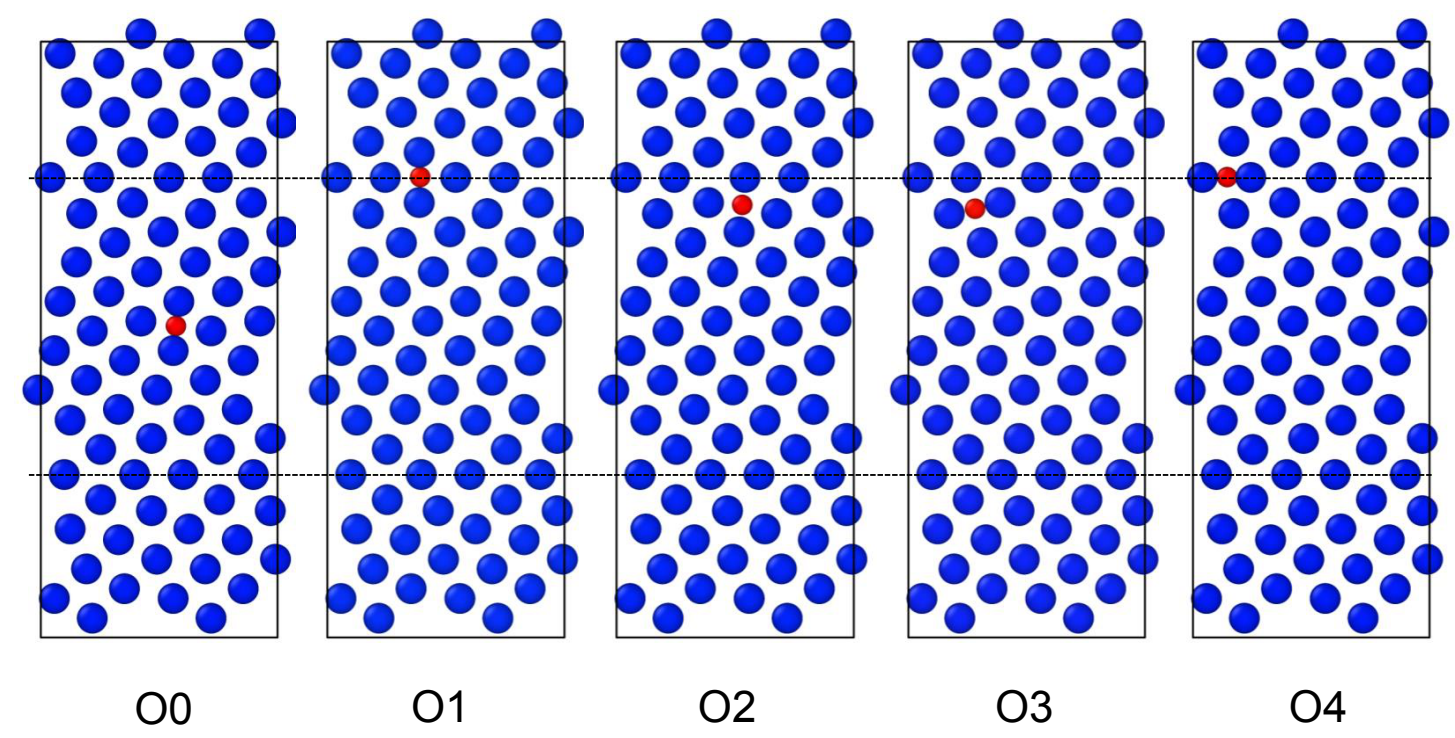

Figure 3: Octahedral interstitial sites O0 through O4, following Ghazisaeidi and Trinkle [16]. The depth of the supercell into the page is $2 a$, approximately $5.90 \AA$, and the twin boundaries are indicated with dashed lines.

Table 1: Formation energy of octahedral interstitials 0 through 4 (O0 - O4, see Figure 3) calculated by DFT and MEAM using the short supercell with a $z$-dimension of $5.90 \mathrm{~A}$.

\begin{tabular}{lccc}
\hline \hline & DFT [16] & DFT (this study) & MEAM (this study) \\
\hline O0 $(\mathrm{eV})$ & 0.0 & 0.0 & 0.0 \\
$\mathrm{O} 1(\mathrm{eV})$ & -0.052 & -0.082 & -0.017 \\
$\mathrm{O} 2(\mathrm{eV})$ & 0.115 & 0.087 & -0.118 \\
$\mathrm{O} 3(\mathrm{eV})$ & 0.193 & 0.177 & 0.077 \\
$\mathrm{O} 4(\mathrm{eV})$ & -0.079 & -0.109 & -0.138 \\
\hline \hline
\end{tabular}


MEAM. Interstitial formation energy and diffusion activation barrier converges as depth of the supercell along the $z$-direction reaches $6 a(17.70 \AA)$ in both the short and tall twin structures. Convergence is achieved in the $x$ - and $y$-directions for the dimensions shown in Figure 1. However, the short cell limits the maximum possible distance from the twin boundary in the $y$-direction to about $8.7 \AA$, restricting our study of the effects of distance of the interstitial from the twin. Based on these results, we employ the tall twin structure with a depth in the $z$-direction of $6 a$ (17.70 $\AA$ ) for MEAM-only calculations and the short twin structure with a depth in the $z$-direction of $2 a(5.90 \AA)$ for calculations utilizing both MEAM and DFT.

\section{The Effects of a Twin Boundary on Interstitial Site Stability and Formation Energy}

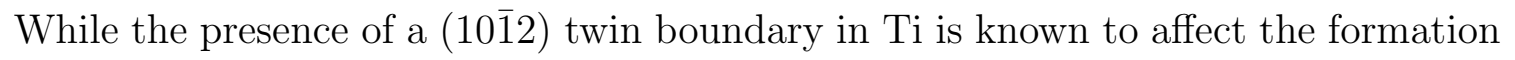
energy of octahedral interstitials in the immediate vicinity of the twin [16], the effects on the hexahedral and crowdion sites, as well as octahedral interstitials further from the twin, have not been previously explored in Ti or any other system. The distribution of $\mathrm{O}$ interstitial sites in the vicinity of a (1012) twin is shown in Figure 4a) with an additional crowdion site located directly into the page behind each of the four octahedral sites. We refer to this distribution of sites as the "0th" layer (0L) from the twin; only unique interstitial sites are shown (unique with respect to both coordination and formation energy), and the remaining sites are obtained by translation and symmetry operations. First, we calculate the formation energy of the 
octahedral, hexahedral, and crowdion interstitials in the 0th layer using both DFT and MEAM and the short supercell with a depth in the $z$-direction of $2 a(5.90 \AA)$. The DFT results provide high quality quantification of how the twin boundary affects formation energy for each interstitial, while the MEAM results provide a further test of the MEAM potential quality and provide insight for interpretation of MEAM results throughout this study. Fully relaxing the system yields the arrangement of sites in Figure $4 \mathrm{~b}$ ); the formation energies relative to the energy of a system with an interstitial in $\mathrm{O} 0$ are shown in Figure 5. The twin boundary has several important effects on the stability and formation energy of the defects. First, the hexahedral site nearest to the twin boundary, H1, and the crowdion site nearest to the twin boundary, C2, relax into stable tetrahedral interstitials, T1 and T2, directly at the twin boundary; both DFT and MEAM predict this behavior, however the MEAM potential produces slightly asymmetrical tetrahedral sites while DFT predicts perfect tetrahedral interstitials. Tetrahedral oxygen interstitials are not stable in bulk $\alpha$-Ti and the (1012) twin therefore introduces a new, previously unreported defect. Second, crowdion interstitials C7 and C8, directly behind octahedral sites O2 and O3, are not stable using DFT or the MEAM; the greater interatomic distances above and below the twin allow the system to relax by moving the twin boundary in response to the presence of these defects. Finally, while DFT predicts that all of the sites in Figure $4 \mathrm{~b}$ ) are stable, crowdion interstitials C3 and C4 are unstable when using the MEAM; this is likely due to the slight overprediction of crowdion interstitial energy and the slight underprediction of diffusion activation barriers for paths leaving the crowdion sites when using this MEAM potential [28], which causes the crowdion sites 

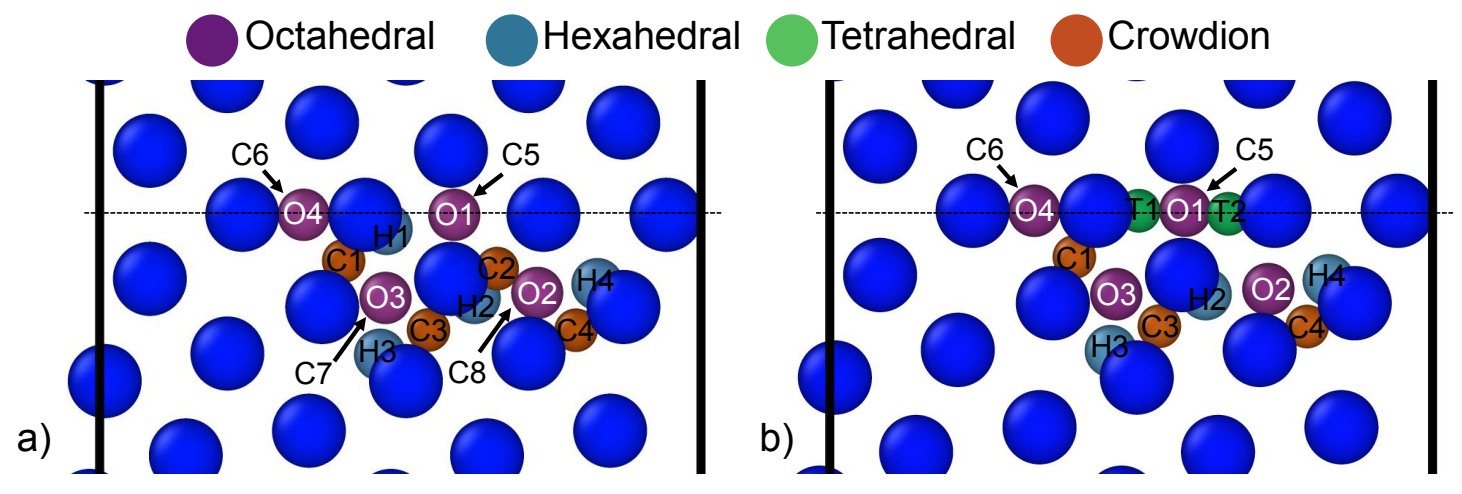

Figure 4: Unique interstitial sites in the 0th layer (0L) from the twin boundary (indicated by a dashed line) a)before and b)after relaxation; the Ti atoms are shown in their unrelaxed positions in both cases for clarity. Crowdion sites C5 - C8 are behind (into the page) octahderal sites O1 - O4, as indicated by arrows. After relaxation, hexahedral $\mathrm{H} 1$ and crowdion $\mathrm{C} 2$ relax to tetrahedral interstitials $\mathrm{T} 1$ and T2, respectively. The crowdion interstitials $\mathrm{C} 7$ and $\mathrm{C} 8$ are unstable.

to be less stable. The energy of these sites is reported in Figure 5 using the peak position of the diffusion pathway through their location, which maintains the correct crowdion structure despite the instability. Overall, the MEAM potential predicts formation energy within $0.2 \mathrm{eV}$ of the DFT values for most interstitials, although in some cases the error is as high as $0.4 \mathrm{eV}$. The VASP and LAMMPS input and output files used to calculate formation energy of each interstitial in the 0L are archived and available [39].

The formation energies of oxygen interstitials continue to differ from the bulk values at greater distance from the (1012) twin boundary. In order to study the effect of distance from the boundary on interstitial formation energy, we identify the interstitial sites matching those shown for the 0L layer in Figure 4 in subsequent layers. Figure 6 shows the position of these interstitials in the 0L layer of the tall supercell 


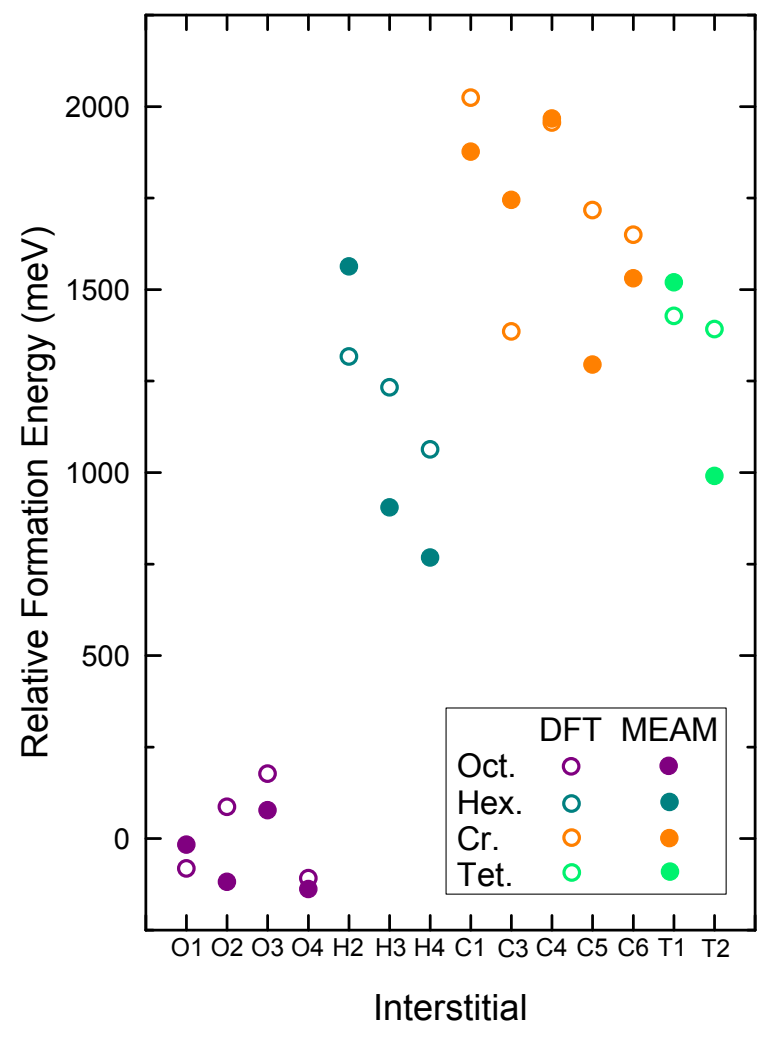

Figure 5: Formation energy of the octahedral, hexahedral, crowdion, and tetrahedral sites in the 0th layer from the twin. Position numbers are provided in Figure 4. 
as well as in the second (2L) and fourth (4L) layers from the twin. Equivalent sites also exist in the first (1L) and third (3L) layers from the twin and are included in our calculations; however the 1L and 3L sites are not shown in Figure 6 for clarity. The change in formation energy of the octahedral, hexahedral, and crowdion interstitials as a function of distance from the twin is shown in Figure $7 \mathrm{a}$ ) -c) as calculated using the MEAM; the tetrahedral interstitial energy for the $0 \mathrm{~L}$ interstitials is included in the hexagonal and crowdion plots to reflect the position that the tetrahedral defect replaces in diffusion paths near the twin. We use the DFT results summarized in Figure 5 to quantify the effect of the twin boundary on interstitial formation energy and the MEAM results in Figure 7 to quantify the range of this effect as a function of distance from the twin boundary. The formation energy of the octahedral defects varies between $+0.177 \mathrm{eV}$ and $-0.109 \mathrm{eV}$ at the twin boundary, as previously reported [16], and returns to bulk-like behavior at a distance of about $10 \AA$ from the twin boundary.

The hexahedral defects behave similarly to the octahedral defects, with the twin boundary modifying formation energy between $+0.097 \mathrm{eV}$ and $-0.157 \mathrm{eV}$. Tetrahedral site $\mathrm{T} 1$, which replaces the hexahedral site nearest to the twin, has a formation energy $0.208 \mathrm{eV}$ higher than the bulk value based on DFT calculations. As with the octahedral interstitials, the hexahedral defects recover bulk like formation energy at a distance of approximately $10 \AA$ from the twin boundary.

The crowdion interstitials behave somewhat differently, with the twin boundary more significantly modifying formation energy between $+0.174 \mathrm{eV}$ and $-0.465 \mathrm{eV}$. Tetrahedral interstitial T2, which replaces a crowdion site nearest to the twin, ex- 
hibits $0.458 \mathrm{eV}$ lower formation energy that a bulk crowdion. The MEAM results in Figure 5 suggest that the effect of the twin boundary on the crowdion interstitials

extends for a greater distance, with some effect still apparent beyond $15 \AA$ from the twin boundary.

Such changes in formation energy are quite large, particularly when considering that occupancy at thermal equilibrium is proportional to $\exp \left(-\Delta E_{f} / k_{B} T\right)$ where $\Delta E_{f}$ is the difference in formation energy of a defect relative to the average energy of the system, $k_{B}$ is the Boltzmann constant, and $T$ is the temperature. Two of the four unique octahedral oxygen interstitials near the twin have a formation energy approximately $100 \mathrm{meV}$ lower than in the bulk, as previously reported [16], and are therefore likely to be occupied; however, given the relatively small distance across which the twin boundary affects formation energy, this change in concentration may be difficult to measure experimentally. The effect of the twin boundary on formation energy is greater for the hexahedral, crowdion, and tetrahedral sites, which are all higher energy when compared to the octahedral sites. As a result, along with increased $\mathrm{O}$ concentration in the immediate vicinity of the twin, a larger fraction of interstitials near the twin will occupy non-octahedral sites.

\section{The Effects of a Twin Boundary on Activation Barriers for Diffusion}

Along with formation energy, the presence of a nearby (1012) twin boundary also affects the activation barriers for diffusion of $\mathrm{O}$ through the lattice. Diffusion of $\mathrm{O}$ in 


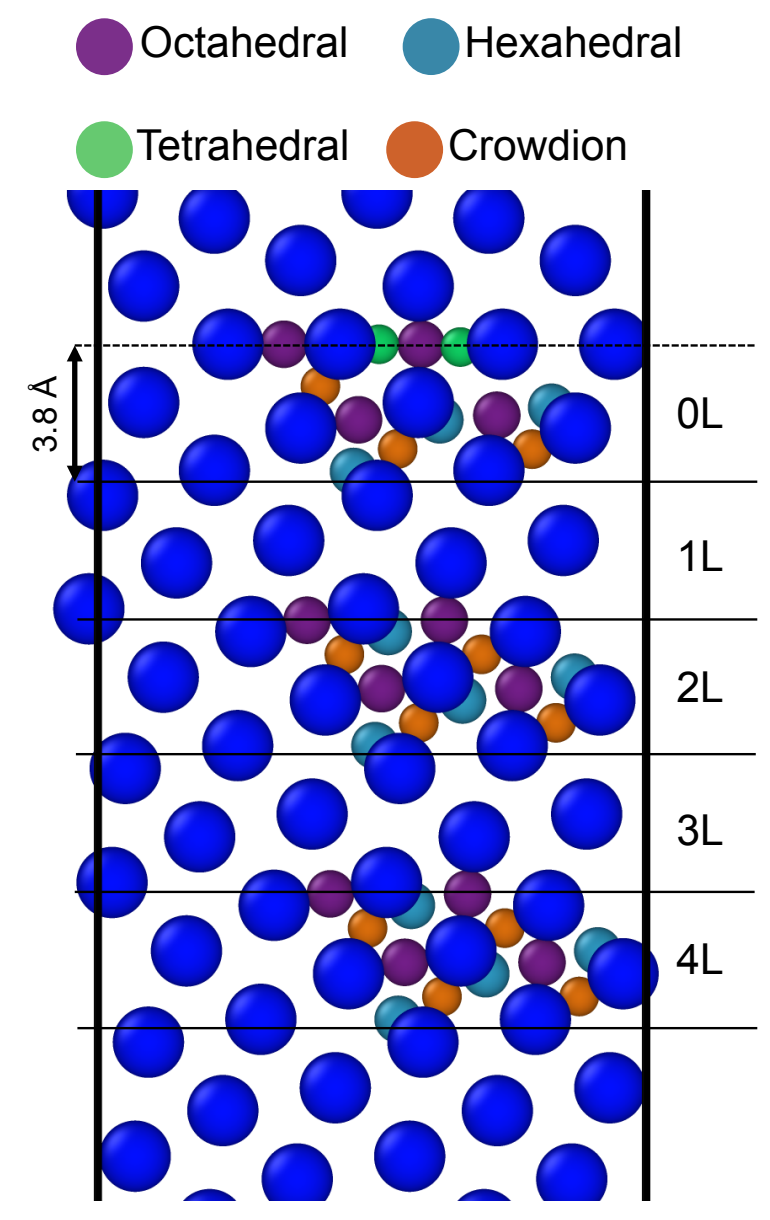

Figure 6: Location of $O$ interstitial sites at increasing distance from a (1012) twin, indicated by a dashed line. The layers are labeled as the 0th layer from the twin (0L) through the fourth layer from the twin (4L). Equivalent sites exist in $1 \mathrm{~L}$ and 3L and have been included in the calculations described here, however they are not shown in the figure for clarity. 


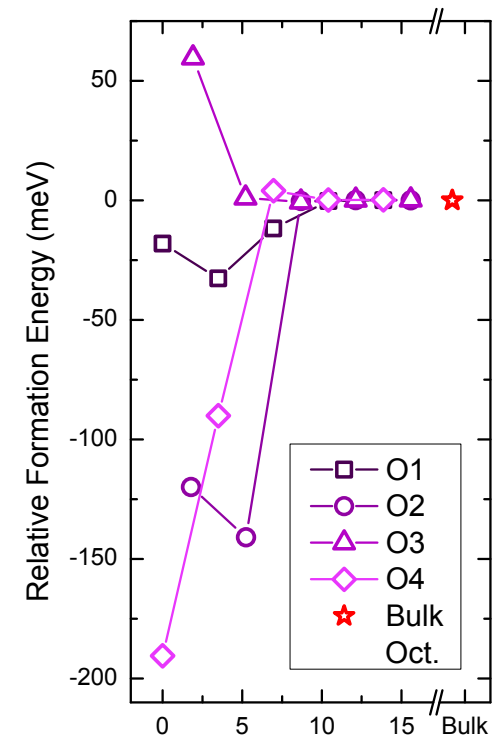

a)

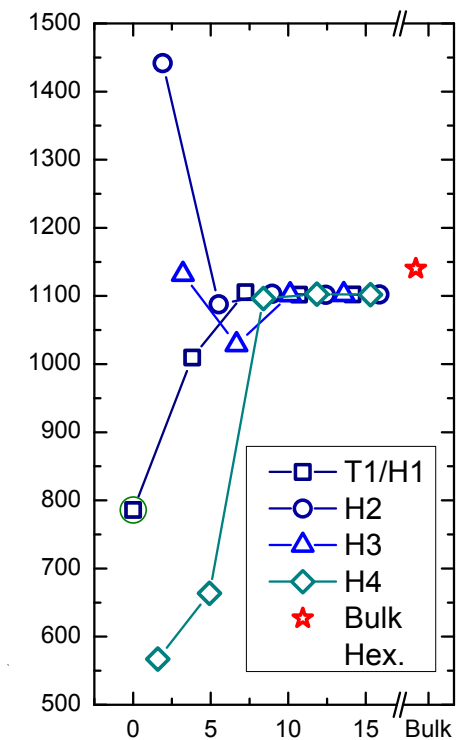

b)

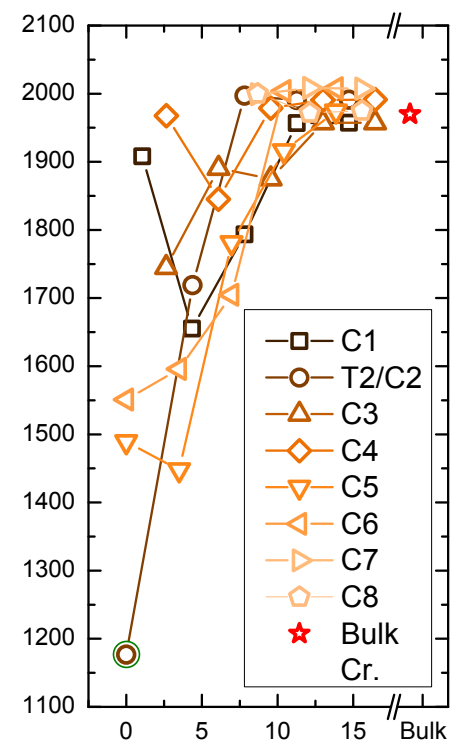

c)

Distance from Twin $(\AA)$

Figure 7: Formation energy of a) octahedral, b) hexahedral, and c) crowdion sites as a function of distance from the twin boundary calculated using the MEAM. The hexahedral and crowdion interstitials which relax into a tetrahedral position in the 0L layer are indicated with a green circle. The bulk formation energy for each defect relative to the octahedral interstitial is shown with a red star. 
bulk $\alpha$-Ti is known to occur by movement between the octahedral, hexahedral, and crowdion interstitial sites with many possible paths contributing to diffusion [14]. There are four independent diffusion paths in the bulk: octahedral $\rightarrow$ octahedral, octahedral $\rightarrow$ hexahedral, octahedral $\rightarrow$ crowdion, and hexahedral $\rightarrow$ crowdion, and the MEAM potential used here is effective for reproducing the activation barriers for these paths [28]. The formation energy results in Figures 5 and 7 demonstrate that there are many more independent diffusion paths near the twin due to the increased number of unique interstitial positions. In order to conduct a complete assessment, we applied the MEAM to calculate the diffusion activation barrier for every possible unique jump in layers 0L and 4L. Figure 8 provides the diffusion activation energy results for each unique path; these results are calculated using the "tall" supercell and therefore the end-point values for each jump differ somewhat from the results shown in Figure 5 which uses the "short" supercell to accommodate DFT calculations.

The $4 \mathrm{~L}$ results are bulk-like and generally collapse onto a single path matching the MEAM predictions of the bulk path. Conversely, the 0L results demonstrate very different behavior near the twin boundary as compared to interstitials in the bulk and $4 \mathrm{~L}$. The nearby (1012) twin boundary has a dramatic effect on some of the octahedral $\rightarrow$ octahedral jumps in 0L, reducing the peak energy by more than 1 $\mathrm{eV}$. While direct octahedral $\rightarrow$ octahedral diffusion does not contribute significantly during bulk diffusion [14], a nearby twin reduces the peak energy of these paths making them comparable to octahedral $\rightarrow$ hexahedral and octahedral $\rightarrow$ crowdion jumps and indicating that direct octahedral $\rightarrow$ octahedral diffusion occurs near the twin. 

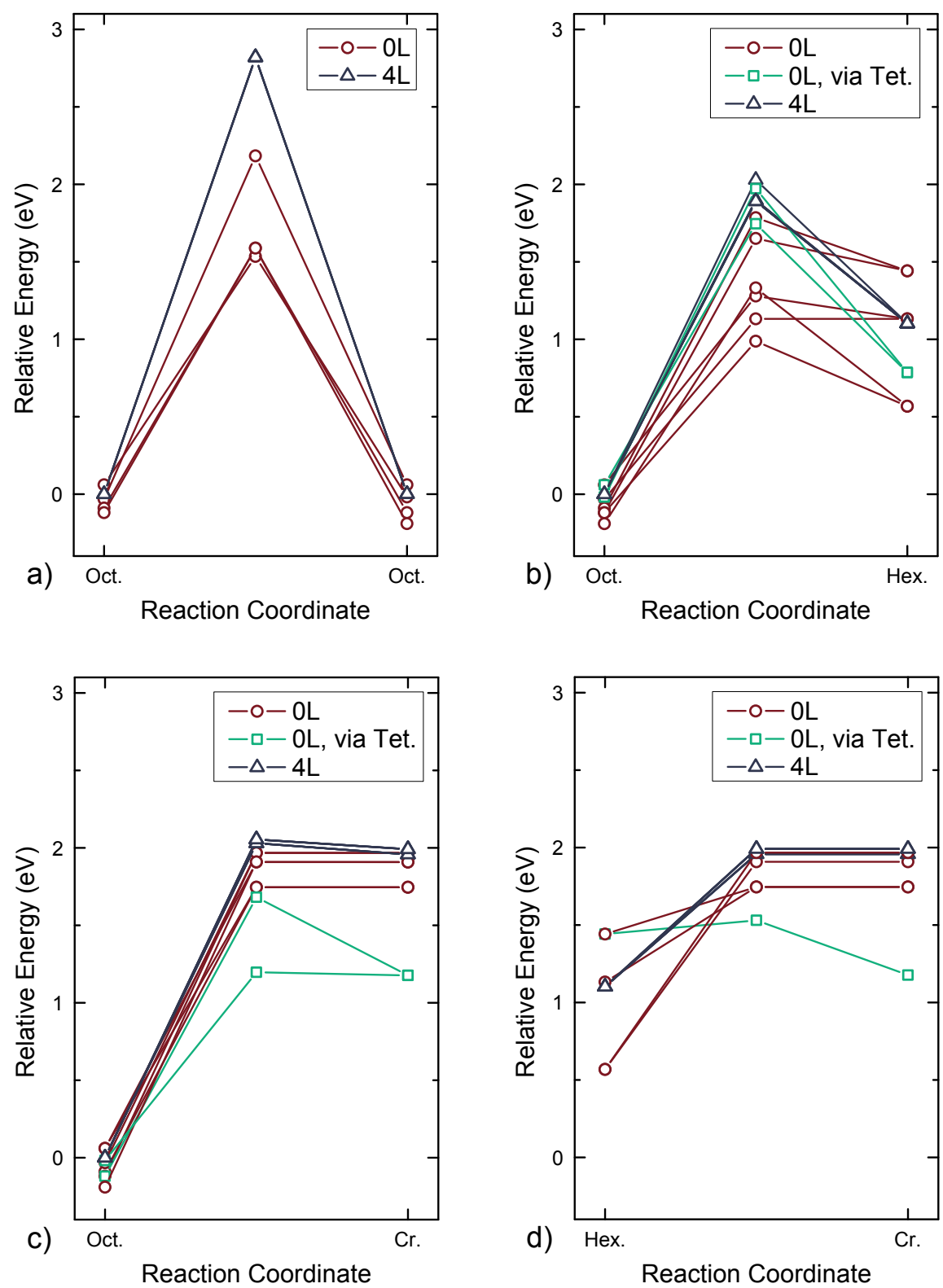

Figure 8: Site energy and activation barriers for diffusion paths in the $4 \mathrm{~L}$ and $0 \mathrm{~L}$ layers in Figure 6. The paths are grouped by the initial and final interstitial characteristics as a) octahedral $\rightarrow$ octahedral, b) octahedral $\rightarrow$ hexahedral, c) octahedral $\rightarrow$ crowdion, and d) hexahedral $\rightarrow$ crowdion. Paths in light green involve a tetrahedral interstitial in the $0 \mathrm{~L}$ layer. 
The peak energy for the octahedral $\rightarrow$ hexahedral jumps is also reduced by up to $1 \mathrm{eV}$ due the nearby twin boundary, however the activation barriers are distributed over a wider range of energies when compared to the octahedral $\rightarrow$ octahedral jumps. The activation barriers for the octahedral $\rightarrow$ tetrahedral jumps in 0L to tetrahedral site T1 (paths highlighted in green in Figure 8b), which relaxes from hexahedral site H1, are not significantly different from the bulk values.

The (1012) twin boundary has a modest effect on the activation barrier of most octahedral $\rightarrow$ crowdion paths in the OL layer. Although the peak energy of some paths is reduced by about $0.4 \mathrm{eV}$, the peak energy for most paths near the twin is only slightly reduced. However, unlike the octahedral $\rightarrow$ hexahedral case, the octahedral $\rightarrow$ tetrahedral jump to tetrahedral site T2 (path highlighted in green in Figure 8c), which relaxes from crowdion site $\mathrm{C} 2$, is significantly lower than other octahedral $\rightarrow$ crowdion paths in the bulk or near the twin. The formation of this tetrahedral interstitial in the twin therefore contributes to diffusion by providing a low energy path across the twin boundary.

Finally, the hexahedral $\rightarrow$ crowdion diffusion paths are also affected by the presence of the twin boundary. The peak energy for most of the paths near the twin is within $0.2 \mathrm{eV}$ of the bulk case. The peak energy of the hexahedral $\rightarrow$ tetrahedral path to tetrahedral site T2 is much lower than the bulk equivalent, similar to the behavior of the octahedral $\rightarrow$ tetrahedral jump to the same site. On the other hand, the tetrahedral $\rightarrow$ crowdion path from $\mathrm{T} 1$ to $\mathrm{C} 1$ is unstable; the peak position relaxes into an neighboring octahedral interstitial and this path therefore does not appear in Figure 8d). 
Having calculated the interstitial site formation energy and diffusion activation barriers for all sites and paths near the twin, we are able to assess the minimum energy pathways for diffusion. In bulk $\alpha$-Ti the activation barrier for octahedral $\rightarrow$ octahedral diffusion is approximately $1 \mathrm{eV}$ higher than the activation barriers for other paths [14, 28] and does not contribute significantly to diffusion. As a result, diffusion in the bulk as well as in the $4 \mathrm{~L}$ layer proceeds by indirect jumps between the low energy octahedral sites via octahedral $\rightarrow$ hexahedral, octahedral $\rightarrow$ crowdion, and hexahedral $\rightarrow$ crowdion diffusion. The combinations of these jumps yield the minimum energy paths far from the twin. Conversely, in the 0L layer there are several octahedral $\rightarrow$ octahedral jumps with activation barriers around $1.5 \mathrm{eV}$ which contribute to diffusion along with the assorted low energy paths via other jumps. We have compiled the five lowest energy paths for a $\mathrm{O}$ interstitial to travel across 0L (to the twin boundary) for comparison with the lowest energy pathways for diffusion in the bulk and 4L. Other diffusion pathways near the twin exist, however the peak energy of the five paths selected here is about $0.3 \mathrm{eV}$ lower than the next lowest energy path and thus this selection represents the most plausible options for O diffusion. Further, the structure is symmetrical about the twin boundary, so a diffusing $\mathrm{O}$ atom will experience the same energy landscape and activation barriers in order to diffuse away from the twin boundary.

A comparison of the lowest energy diffusion pathways across the twin with the lowest energy diffusion pathways in the bulk is provided in Figure 9. The peak energy of the bulk paths varies from $1.97 \mathrm{eV}$ to $2.02 \mathrm{eV}$, closely aligned with both experimental [13] and DFT [14] results. The peak energy for diffusion across the 
0L layer (and thus to, and across the twin boundary) varies from $1.53 \mathrm{eV}$ to 1.68 $\mathrm{eV}$, a reduction of $0.29-0.49 \mathrm{eV}$. Diffusivity scales with $\exp \left(-\Delta E_{B} / k_{B} T\right)$, where $E_{B}$ is the activation barrier energy, and a change in peak energy of this magnitude therefore has a large impact on the rate of $\mathrm{O}$ diffusion near and across the twin.

Unimpeded, twin growth is observed to occur at rates approaching the speed of sound in hcp metals (for example, [43, 44]). Hence despite accelerated kinetics, O diffusion near the twin is still comparatively slow and twin growth rate may be decreased by the presence of $\mathrm{O}$. Compared to the activation barrier for $\mathrm{O}$ diffusion in the bulk, the reduced activation barrier for $\mathrm{O}$ diffusion across the twin boundary is closer to the experimentally measured activation energy for twin growth [11, 12]; however, the activation barriers for these processes still differ by almost $1 \mathrm{eV}$, and thus the interaction of twins and $\mathrm{O}$ interstitials likely occurs via a more complicated mechanism than serial twin boundary motion/oxygen diffusion. Nonetheless, the trends observed here suggest that the interaction mechanisms merit further study. 


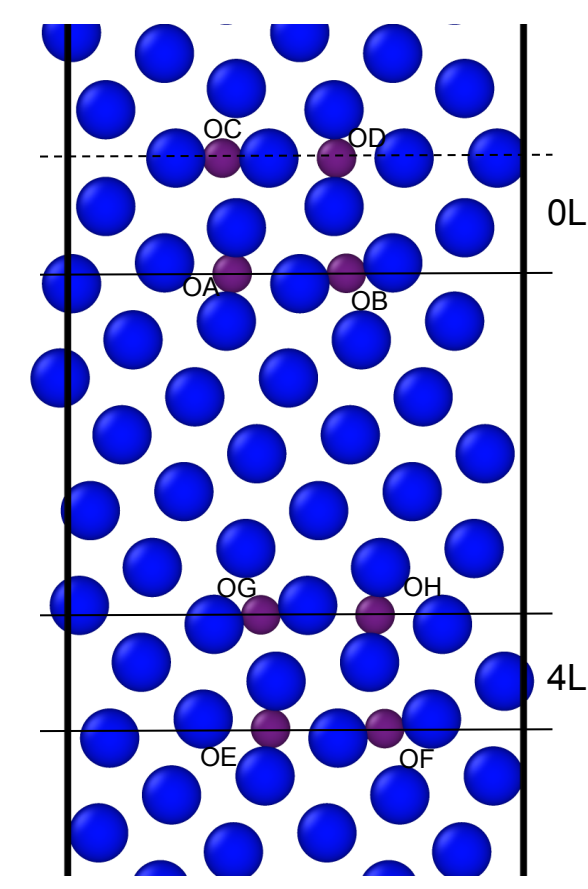

a)
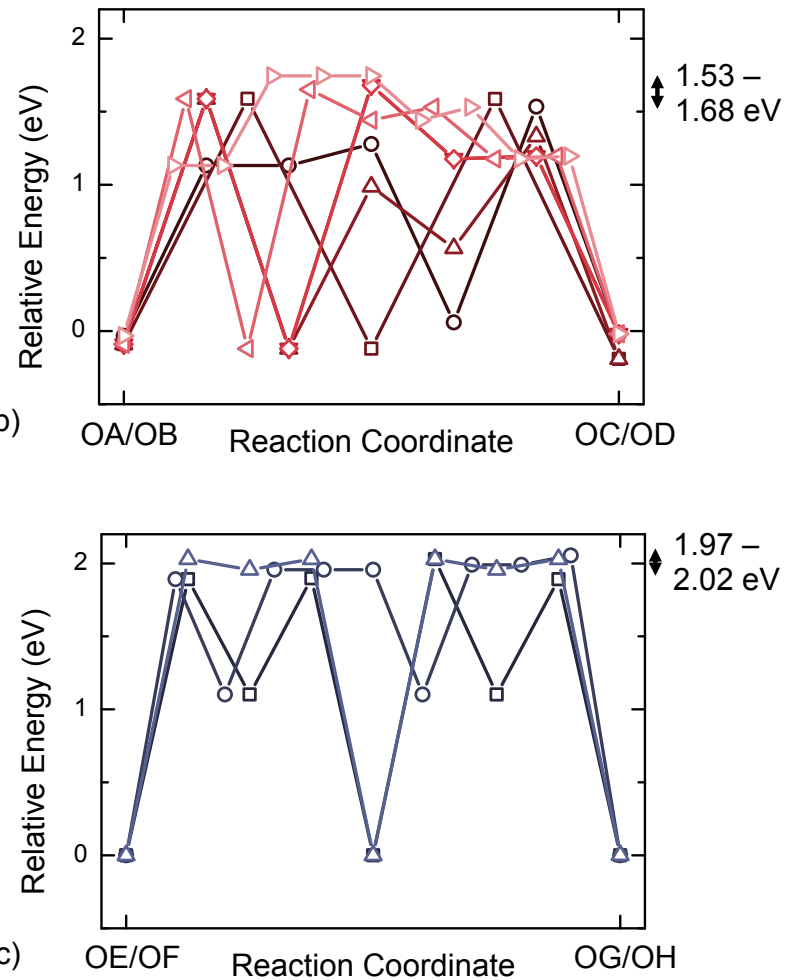

Figure 9: The minimum energy pathways for diffusion of oxygen between octahedral sites spanning the 0th layer from the twin (0L) and fourth layer from the twin $(4 \mathrm{~L})$ as shown in a) with the twin boundary indicated by a dashed line. An oxygen atom diffusing along the lowest energy pathways in b) OL must overcome lower peak activation energies for diffusion (shown to the right of each plot) when compared to an oxygen atom diffusing along the lowest energy pathways in c) $4 \mathrm{~L}$.

\section{Conclusion}

The interactions between alloying or impurity elements and twin growth are not well understood, despite opportunities for performance improvements across the many 
hcp metals where deformation twinning is prevalent. In the case of $\alpha$-Ti, experimental evidence suggests that $\mathrm{O}$ interstitials could result in time-dependent twinning by interfering with the twin growth process. As a first step towards understanding the mechanisms of these interactions, we have applied a combination of DFT and MEAM calculations to assess the effect of a (101̄2) twin boundary on the formation energy of all unique $\mathrm{O}$ interstitial sites along with the diffusion activation barriers between the sites.

The formation energy of octahedral, hexahedral, and crowdion $\mathrm{O}$ interstitials is modified by as much as $0.465 \mathrm{eV}$ near the twin boundary and returns to bulk-like values at a distance of about $10 \AA$ from the twin. Two unique tetrahedral interstitials are stable in the twin boundary, despite being unstable in the bulk, with formation energies comparable to hexahedral interstitials. Two of the four unique octahedral oxygen interstitials near the twin have a reduced (more negative) formation energy and are therefore likely to be occupied, with an increase in $\mathrm{O}$ concentration extending to about $10 \AA$ from the twin boundary. The twin boundary has a greater effect on the formation energy of the hexahedral, crowdion, and tetrahedral sites, which are all higher energy when compared to the octahedral sites. As a result, along with increased $\mathrm{O}$ concentration in the immediate vicinity of the twin, a larger fraction of interstitials near the twin will occupy non-octahedral sites. While the short range of the increased concentration may make experimental measurement difficult, the increased concentration of $\mathrm{O}$ interstitials near the twin boundary further indicates that twin/O interactions are plausible.

The diffusion activation barriers for $\mathrm{O}$ near the (1012) twin boundary are uni- 
formly lower than in the bulk. In some cases the barriers are reduced by more than 1 $\mathrm{eV}$ due to a nearby twin boundary, creating many low energy diffusion pathways. Of the two unique tetrahedral interstitials in the twin boundary, only one contributes significantly to $\mathrm{O}$ diffusion while the other does not connect to a low energy diffusion path. Our MEAM calculations indicate that the peak energy for diffusion of $\mathrm{O}$ through bulk Ti is about $2 \mathrm{eV}$, corresponding well to measured and DFT-calculated values. After reviewing the available paths for an $\mathrm{O}$ interstitial to move to and across a twin boundary, we find that the peak energy for diffusion is between 1.53 $\mathrm{eV}$ and $1.68 \mathrm{eV}$. Such a large reduction in peak energy indicates that $\mathrm{O}$ diffusion occurs much more readily across a twin boundary when compared to the bulk. However, accelerated $\mathrm{O}$ diffusion near the twin is still much slower than unimpeded twin growth and $\mathrm{O}$ is likely to play a role in the observed strain-rate and time dependence of (1012) twinning. That our newly reported activation barriers for diffusion near a (1012) twin are still higher than the measured activation energy of twin growth indicates that the mechanisms of O-twin interaction are more complicated than a serial growth/diffusion process and merit further investigation.

\section{Acknowledgments}

We are very grateful for assistance from Dr. Roman Tsyshevskiy and Dr. R. Prakash Kolli during this work and in preparation of the manuscript. We appreciate access to and use of resources of the National Energy Research Scientific Computing Center (NERSC), which is supported by the Office of Science of the U.S. Department of 
Energy under Contract No. DE-AC02-05CH11231. We acknowledge the University of Maryland supercomputing resources (http://www.it.umd.edu/hpcc) made available in conducting the research reported in this research. Portions of this work were supported by the National Science Foundation under Grant No. DMR-0906994.

\section{References}

[1] G. Lutjering and J. Williams, Titanium. Berlin, New York: Springer, 2 ed., 2007.

[2] M. Peters, J. Kumpfert, C. H. Ward, and C. Leyens, "Titanium alloys for aerospace applications," Adv. Eng. Mat., vol. 5, no. 6, pp. 419-427, 2003.

[3] C. Elias, J. Lima, R. Valiev, and M. Meyers, "Biomedical applications of titanium and its alloys," JOM, vol. 60, no. 3, pp. 46-49, 2008.

[4] R. Schutz, C. Baxter, P. Boster, and F. Fores, "Applying titanium alloys in drilling and offshore production systems," JOM, vol. 53, no. 4, pp. 33-35, 2001.

[5] R. A. Lindemann and C. J. Voorhees, "Mars exploration rover mobility assembly design, test and performance," in Systems, Man and Cybernetics, 2005 IEEE International Conference on, vol. 1, pp. 450-455, IEEE, 2005.

[6] J. Murray and H. Wriedt, "The O-Ti (oxygen-titanium) system," J. Phase Equilib., vol. 8, no. 2, pp. 148-165, 1987. 
[7] S. Ankem, C. Greene, and S. Singh, "Time dependent twinning during ambient temperature creep of $\alpha$ Ti-Mn alloy," Scripta Met. Mat., vol. 30, no. 6, pp. 803808, 1994.

[8] M. Biget and G. Saada, "Effect of interstitial impurities on twinning of titanium and zirconium," J. de Physique III, vol. 5, no. 11, pp. 1833-1840, 1995.

[9] P. Oberson and S. Ankem, "Why twins do not grow at the speed of sound all the time," Phys. Rev. Lett., vol. 95, no. 16, p. 165501, 2005.

[10] A. Aiyangar, B. Neuberger, P. Oberson, and S. Ankem, "The effects of stress level and grain size on the ambient temperature creep deformation behavior of an alpha Ti-1.6 wt pet V alloy," Met. Trans. A, vol. 36, pp. 637-644, 2005.

[11] P. Oberson, Z. Wyatt, and S. Ankem, "Modeling interstitial diffusion controlled twinning in alpha titanium during low-temperature creep," Scripta Mat., vol. 65, no. 7, pp. 638-641, 2011.

[12] Z. Wyatt, W. Joost, D. Zhu, and S. Ankem, "Deformation mechanisms and kinetics of time-dependent twinning in an $\alpha$-titanium alloy," International Journal of Plasticity, vol. 39, pp. 119-131, 2012.

[13] F. Bregolin, M. Behar, and F. Dyment, "Diffusion study of 18-O implanted into $\alpha$-Ti using the nuclear resonance technique," Appl. Phys. A, vol. 86, no. 4, pp. 481-484, 2007.

[14] H. H. Wu and D. R. Trinkle, "Direct diffusion through interpenetrating networks: Oxygen in titanium," Phys. Rev. Lett., vol. 107, no. 4, p. 045504, 2011. 
[15] Z. Liu and G. Welsch, "Literature survey on diffusivities of oxygen, aluminum, and vanadium in alpha titanium, beta titanium, and in rutile," Met. Trans. A, vol. 19 , no. 4, pp. 1121-1125, 1988.

[16] M. Ghazisaeidi and D. Trinkle, "Interaction of oxygen interstitials with lattice faults in Ti," Acta Mat., vol. 76, pp. 82-86, 2014.

[17] M. Bhatia, X. Zhang, M. Azarnoush, G. Lu, and K. Solanki, "Effects of oxygen on prismatic faults in $\alpha$-Ti: a combined quantum mechanics/molecular mechanics study," Scripta Mat., vol. 98, pp. 32-35, 2015.

[18] G. Kresse and J. Hafner, "Ab initio molecular dynamics for liquid metals," Phys. Rev. B, vol. 47, pp. 558-561, Jan 1993.

[19] G. Kresse and J. Hafner, "Ab initio molecular-dynamics simulation of the liquid-metal-amorphous-semiconductor transition in germanium," Phys. Rev. B, vol. 49, pp. 14251-14269, May 1994.

[20] G. Kresse and J. Furthmüller, "Efficiency of ab-initio total energy calculations for metals and semiconductors using a plane-wave basis set," Comput. Mat. Sci., vol. 6, no. 1, pp. 15-50, 1996.

[21] G. Kresse and J. Furthmüller, "Efficient iterative schemes for ab initio totalenergy calculations using a plane-wave basis set," Phys. Rev. B, vol. 54, pp. 11169-11186, Oct 1996.

[22] P. E. Blöchl, "Projector augmented-wave method," Phys. Rev. B, vol. 50, pp. 17953-17979, Dec 1994. 
[23] G. Kresse and D. Joubert, "From ultrasoft pseudopotentials to the projector augmented-wave method," Phys. Rev. B, vol. 59, pp. 1758-1775, Jan 1999.

[24] J. P. Perdew, K. Burke, and M. Ernzerhof, "Generalized gradient approximation made simple," Phys. Rev. Lett., vol. 77, pp. 3865-3868, Oct 1996.

[25] S. Plimpton, "Fast parallel algorithms for short-range molecular dynamics," $J$. Comput. Phys., vol. 117, no. 1, pp. 1-19, 1995.

[26] Y.-M. Kim, B.-J. Lee, and M. Baskes, "Modified embedded-atom method interatomic potentials for Ti and Zr," Phys. Rev. B, vol. 74, no. 1, p. 014101, 2006.

[27] M. Baskes, "Modified embedded atom method calculations of interfaces," Tech. Rep. SAND-96-8484C; CONF-9603153-1, Sandia National Laboratories Livermore, USA, 1996. http://www.osti.gov/scitech/biblio/224267.

[28] W. Joost, S. Ankem, and M. Kuklja, "A modified embedded atom method potential for the titanium-oxygen system," Mod. Sim. in Mat. Sci. and Eng., vol. 23, no. 1, p. 015006, 2015.

[29] B.-J. Lee and M. Baskes, "Second nearest-neighbor modified embedded-atommethod potential," Phys. Rev. B, vol. 62, no. 13, p. 8564, 2000.

[30] B.-J. Lee, M. Baskes, H. Kim, and Y. K. Cho, "Second nearest-neighbor modified embedded atom method potentials for BCC transition metals," Phys. Rev. B, vol. 64, no. 18, p. 184102, 2001. 
[31] M. I. Baskes, "Application of the embedded-atom method to covalent materials: A semiempirical potential for silicon," Phys. Rev. Lett., vol. 59, pp. 2666-2669, Dec 1987.

[32] M. I. Baskes, J. S. Nelson, and A. F. Wright, "Semiempirical modified embedded-atom potentials for silicon and germanium," Phys. Rev. B, vol. 40, pp. 6085-6100, Sep 1989.

[33] M. Baskes, "Modified embedded-atom potentials for cubic materials and impurities," Phys. Rev. B, vol. 46, pp. 2727-2742, Aug 1992.

[34] M. Baskes and R. Johnson, "Modified embedded atom potentials for HCP metals," Modell. Simul. Mater. Sci. Eng., vol. 2, no. 1, p. 147, 1994.

[35] G. Henkelman, B. P. Uberuaga, and H. Jónsson, "A climbing image nudged elastic band method for finding saddle points and minimum energy paths," $J$. Chem. Phys., vol. 113, p. 9901, 2000.

[36] G. Henkelman and H. Jónsson, "Improved tangent estimate in the nudged elastic band method for finding minimum energy paths and saddle points," J. Chem. Phys., vol. 113, p. 9978, 2000.

[37] D. Sheppard, P. Xiao, W. Chemelewski, D. D. Johnson, and G. Henkelman, "A generalized solid-state nudged elastic band method," J. Chem. Phys., vol. 136, p. $074103,2012$. 
[38] A. Stukowski, "Visualization and analysis of atomistic simulation data with OVITO-the open visualization tool," Modelling and Simulation in Materials Science and Engineering, vol. 18, no. 1, p. 015012, 2010.

[39] http://hdl.handle.net/11256/272.

[40] G. Welsch, R. Boyer, and E. Collings, Materials properties handbook: titanium alloys. ASM international, 1993.

[41] T. Braisaz, P. Ruterana, G. Nouet, and A. Serra, "High-resolution electron microscopy study of the (1012) twin and defects analysis in deformed polycrystalline alpha titanium," Phil. Mag. Lett., vol. 74, no. 5, pp. 331-338, 1996.

[42] J. R. Morris, Y. Ye, and M. H. Yoo, "First-principles examination of the twin boundary in hcp metals," Philosophical Magazine, vol. 85, no. 2-3, pp. 233-238, 2005.

[43] R. Bunshah, "Rates of deformation twinning in metals," in Proceedings of the Conference on Deformation Twinning (R. Reed-Hill, J. Hirth, and H. Rogers, eds.), pp. 390-392, TMS, 1964.

[44] M. V. Klassen-Neklyudova, Mechanical twinning of crystals. New York: Consultants Bureau, 1964. 


\section{Graphical Abstract}

Twinning is an important deformation mechanism in many hexagonal close packed metals, including $\alpha$-titanium (Ti) alloys. However, the mechanisms for twin nucleation, growth, and interaction with other defects are not completely understood. In this study we interrogate the behavior of oxygen $(\mathrm{O})$ interstitials near a $(10 \overline{1} 2)$ twin boundary using a combination of density functional theory (DFT) and modified embedded atom method (MEAM) calculations. The presence of the twin boundary significantly affects both interstitial formation energy as well as the activation barriers for diffusion between sites. We demonstrate that a tetrahedral interstitial is stable in the twin boundary, despite being unstable in bulk Ti, while the formation energies of the octahedral, hexahedral, and crowdion interstitials are all modified by a nearby twin. Further, the activation barriers for diffusion in the region near the twin are uniformly lower than in the bulk. An atom diffusing across the twin boundary moves through several paths with peak energies more than $0.3 \mathrm{eV}$ lower than for comparable diffusion far from the twin, suggesting that the (1012) twin is a fast diffusion pathway and movement of oxygen interstitials across the twin during twin growth is possible.

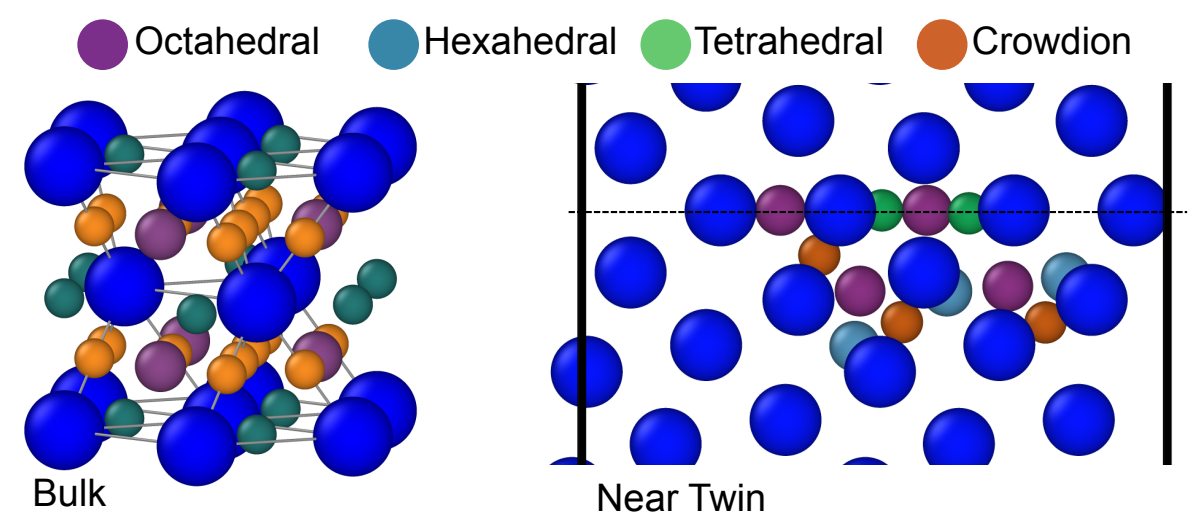

